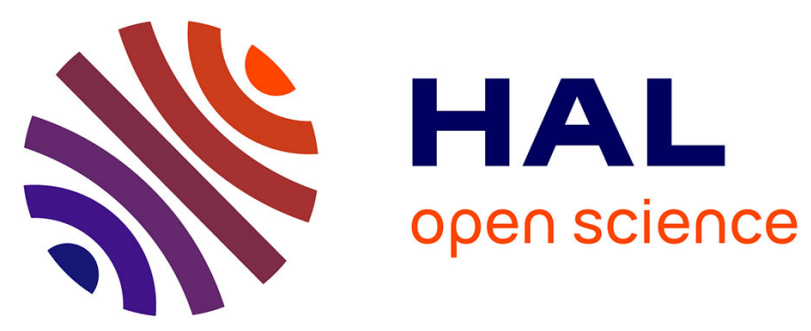

\title{
DRACeo: A smart simulator to deploy energy saving methods in microservices based networks
}

Hernán H Álvarez Valera, Marc Dalmau, Philippe Roose, Christina Herzog, Jorge Larracoechea

\section{To cite this version:}

Hernán H Álvarez Valera, Marc Dalmau, Philippe Roose, Christina Herzog, Jorge Larracoechea. DRACeo: A smart simulator to deploy energy saving methods in microservices based networks. WETICE, Jun 2020, Bayonne, France. hal-03111199

\section{HAL Id: hal-03111199 https://hal-univ-pau.archives-ouvertes.fr/hal-03111199}

Submitted on 15 Jan 2021

HAL is a multi-disciplinary open access archive for the deposit and dissemination of scientific research documents, whether they are published or not. The documents may come from teaching and research institutions in France or abroad, or from public or private research centers.
L'archive ouverte pluridisciplinaire HAL, est destinée au dépôt et à la diffusion de documents scientifiques de niveau recherche, publiés ou non, émanant des établissements d'enseignement et de recherche français ou étrangers, des laboratoires publics ou privés. 


\title{
DRACeo: A smart simulator to deploy energy saving methods in microservices based networks
}

\author{
Hernan Humberto Alvarez Valera*, Marc Dalmau ${ }^{\dagger}$, Philippe Roose ${ }^{\ddagger},{ }^{\uparrow}$ Jorge Larracoechea and ${ }^{\S}$ Christina Herzog \\ $* \dagger \ddagger$ I E2S UPPA, University of Pau \\ 64600 Anglet / FRANCE \\ Email: *hhavalera@univ-pau.fr, ${ }^{\dagger}$ Marc.Dalmau@iutbayonne.univ-pau.fr, ${ }^{\ddagger}$ Philippe.Roose@iutbayonne.univ-pau.fr, \\ Ijorge-andres.larracoechea@etud.univ-pau.fr, ${ }^{\S}$ herzog@efficit.com \\ $\S_{\text {EFFICIT SAS - Mauzac, France }}$
}

\begin{abstract}
Nowadays, many researchers work to identify microservices-based application deployments and scheduling solutions to save energy without decreasing functional QoS. In this work, we present DRACeo: A simulator that allows facing this challenge in a simple and efficient way, enabling its users to focus uniquely on microservices deployment/scheduling algorithms and its hardware/software repercussions (load vs. energy consumption) without worrying about low-level network configurations or operating system issues. DRACeo is able to deploy and schedule (move, duplicate, start/stop) microservices and their dependencies on various devices with software and hardware heterogeneity (CPU, bandwidth, RAM, Battery, etc.), taking into account various scheduling heuristics algorithms: centralized vs noncentralized. To do this, DRACeo allows deploying custom network topologies based on client-server schemes or p2p distributions, where devices can (dis)appear, turn on/off obeying random circumstances or user strategies. Finally, the simulator performs relevant operations such as QoS definition, resource monitoring, calculation of energy saved and consumption tracking (at device and network level). We tested some ideas based on our previous work "Kaligreen" to demonstrate the effectiveness of DRACeo.

Index Terms-microservices, middleware, energy, consumption, CPU, network, hard disk, prototype, simulator
\end{abstract}

\section{INTRODUCTION}

Currently, many companies and scientists use microservices because they allow architectural advantages such as acceleration of deployment cycles, modularity, improvement of maintainability, high availability, scalability, etc [1]. For example, Docker and Kubernetes [2] manage microservices on the cloud or even on grid environments, while Kalimucho [3] does the same on user device level.

Then, to manage them correctly and to achieve a desired QoS (i.e. performance and response time), it is necessary to face several issues such as, load balancing, scalability, etc. Some centralized approaches, such as the Netflix Conductor [4], help addressing them by managing microservices' connection aspects; whereas decentralized approaches like Kalimucho [3], address these issues by allowing the development of in-device algorithms for each node to perform microservices movement or duplication operations. Then, knowing that both (and also hybrid) approaches have advantages and disadvantages; we find it useful to have a tool that allows working with these two approaches. Kalimucho middleware [3] enables each device to manage microservices and their connections, allowing to deploy both types of approaches. It deploys and operates (start, stop, move and duplicate) software components from one device to another, regardless of whether the device belongs to cloud entities or user terminals. Then, as Kalimucho is able to measure on each device CPU activity, current bandwidth and RAM availability, it is a good middleware candidate for energy savings purposes. For this reason, in some of our previous works [5] [6] we proposed a middleware called "Kaligreen" strongly inspired by Kalimucho. Kaligreen proposes that each device in the network has a supervisory entity which: (1) constantly monitors the device energy situation (i.e. battery, energy consumption, etc.) and the load of its hardware components; (2) classifies microservices according to their type and features [6] and (3) executes its own scheduling algorithm based on smart negotiations [5]. Then, while we did a POC to prove that moving/duplicating services in a distributed architecture can save energy, we did not study enough the heterogeneity of devices or the capabilities that each hardware component offers. Nor did we consistently consider the QoS of applications when microservices are scheduled at different types and levels of network topologies. For these reasons, we propose DRACeo: A tool that allows evaluating different heuristics of microservice deployment/scheduling and its repercussions taking into account: (1) the capabilities and particular characteristics of each hardware component, (2) the simple configuration, through a GUI or an API, of a centralized (i.e. client server), decentralized (i.e. p2p architectures [7]) or hybrid network configuration and (3) the definition and evaluation of QoS, energy metrics and quantity of operations quantity (movements, duplications, deployments and deletions) when a scheduling algorithm is executed. Thereby, DRACeo is able to virtualize an environment controlled by any middleware that deploys and schedules microservices, allowing researchers to create any type of scheduling algorithm and measure its consequences in terms of energy and application efficiency.

This article presents related works in section II, the policies and the architecture of the simulator in Section III and finally, in section IV, some examples and results are shown.

\section{RELATED WORKS}

In order to understand and formally express the set of consequences in terms of application efficiency and energy 
consumption in any distributed environment, it is necessary to have a tool that allows modeling multiple scenarios and implementing various deployment and scheduling algorithms. Packet oriented simulators [8] [9] [10] [11] [12], for example, allow to evaluate network traffic as well as the result of using certain transmission protocols. They also allow modeling scenarios to evaluate data streaming techniques and features, latency, cases of data loss and data duplication and even modeling a specialized Information Centric Networking (ICN) [13] architecture, among others. However, we consider that these tools cannot replicate the behavior (i.e. use of resources, application QoS and energy consumption) of a service-based architecture, where a higher level of abstraction is needed. On the other hand, services architecture based simulators [14] [15] [16] [17] [18] [19] enable performing a different set of operations such as: services management, monitoring the exchange of information [18], adding virtualization policies [14] and observing energy and QoS repercussions. They also provide other metrics such as: latency, requests [16], resources availability and power consumption. Then, even though these simulators are competent in analyzing services-based scenarios, we consider they do not offer a simple and transparent way for deploying high-level distributed/centralized scheduling algorithms among heterogeneous devices belonging to different network topologies in which, for example, the relation between energy consumption analysis and a defined QoS is important.

For all these reasons, DRACeo is strongly focused on the application of both centralized and distributed scheduling algorithms, allowing to analyze at deployment/execution time: (1) the definition, measurement and modelization of applications QoS, (2) the load of each node's hardware components and (3) the energy consumption of a particular device as well as of the entire modeled network. Furthermore, DRACeo allows microservices orchestration and choreography by reconfiguring microservice's connections and hosting devices.

\section{DRACEO SimULATOR}

DRACeo is a desktop and portable simulator, able to manage (deploy/schedule) any microservice based application in any network architecture (non-centralized, centralized and hybrid). Then, in our simulator context, an application is defined as a directed graph whose nodes are microservices and edges the connections between them. Furthermore, every application is deployed on a graph of connected devices, where the nodes could be devices with heterogeneous characteristics or abstract entities such as clusters or cloudlets. Device graph edges are the network connections that exist between each node (ethernet, wireless, $4 \mathrm{~g}$, or bluetooth), which allow to find the transfer rate at which the microservices can send or receive data to each other. Then, to understand the interaction of both graphs, it is necessary to describe their nodes and edges in detail, which are the entities of our simulator:

\section{A. Simulator entities}

1) Device: Processing entity identifiable by a unique ID. The simulator by default supports desktop computers, laptops and smartphones; however, users are also able to configure their own type of device. Each one of these has different components capabilities in terms of CPU frequency (presence or absence of PCPG and DVFS are also included), RAM size, hard drive speed, network transfer rate and battery (optional), depending on which type they are or what custom configuration was performed. Each device is capable of executing microservices, providing them a quantity of resources according to their own needs and those of other competitors microservices (like proportional share scheduling; however we are currently implementing other philosophies as round robin or a simplified version of CFS [20], which uses a red and black tree to organize processes). In addition, each device has a supervisor service running on it [5]. It allows (1) tracking the status of each of its components in terms of load/capacity/energy consumption and (2) executing a distributed scheduling algorithm (i.e. move or duplicate a microservice to another known or reachable device, considering negotiation criteria, load balancing, number of hops, etc.)

2) Microservice: Functional entity identifiable by a unique ID, which has a defined and precise function (i.e. a code that represents a function and that allows to be duplicated by the simulator as explained in the next section III-B). When deployed on a device, the microservice claims for a certain amount of resources in terms of CPU, network, RAM and disk. DRACeo supports by default 3 types of microservices: graphical user interface microservices, calculation microservices and data management microservices. The only differences between them are their default resources consumption, the amount of data they send/receive, their size and the restriction of being moved or not from one device to another. For example, a UI microservice may not be moved (since the user experience would be affected); while a computing microservice may be duplicated or moved to another nearby device. A data management microservice can be moved, but may have a strong impact on bandwidth consumption. As with devices, a user has the ability to create its own type of microservices.

3) Connection: DRACeo defines and manages two kinds of connections: physical connections that exist between devices and logical/functional connections between microservices. A physical connection logically supports many microservices connections and enables to track the current and maximum possible transfer rate (i.e. between 2 devices: the maximum transmission capacity of the device with the network interface with less transmission capacity). Then, this allows understanding: (1) the general state (i.e. at energy and load level) of a given device network interface, even if it belongs to abstract entities such as clusters, cloudlets, etc. and (2) the set of microservice connections status (i.e. expected transmission rate vs. real) that use a physical connection. Furthermore, connections between microservices logically store the set of physical connections they use to communicate. That is, it is possible to know, through the supervisor microservice, the path of devices a microservice connection uses to work, as well as the current and expected transfer rate. 
4) Application: A directed graph of microservices identifiable by an ID. Each microservice (node) can have connections (edges) as dependencies relations with several other microservices which run on different devices on the network.

5) Operations center: Centralized entity which stores all references to connections and existing devices of a deployed scenario. In this way, any type of middleware can be configured and any centralized scheduling algorithm can be applied.

6) Abstract entities: Resources providers such as cloudlets and clusters. Both can be deployed and connected in the same way as devices, establishing the amount of resources to offer and manage. Therefore, our simulator is compatible with both, small networks of user devices and cloud networking.

\section{B. Simulator Operations}

DRACeo is able to apply different types of heuristics for microservices (re)deployment and scheduling through a network. For this, the following operations have been implemented:

1) Device deployment: As previously mentioned, a user can deploy/declare default DRACeo devices (i.e. desktop computer, laptop and smartphone); however, he can also deploy "custom" devices, specifying their capabilities in terms of (1) CPU frequency in Ghz, (2)RAM capacity in MB, (3) network capacity in $\mathrm{Mb} / \mathrm{s}$, (4) hard drive in $\mathrm{MB} / \mathrm{s}$ and (5) battery specification. Furthermore, each time a device is deployed, the user must specify an $\mathrm{x}, \mathrm{y}$ position on a Cartesian plane of metric units. This possibility allows studying deployment techniques based on distance heuristics or techniques to improve routes.

2) Device suppression: DRACeo allows the suppression of devices (1) Manually, by the user's decision and (2) automatically, according to the deployed scenario. A user can activate a "disappear" option for each device, causing the simulator to delete the device after a random, established or battery related time; simulating unexpected connection loss.

3) Microservice deployment: DRACeo allows simulating the execution of microservices on devices. This means that once deployed, a microservice uses a quantity of resources for a determined time or indefinitely [6]. This time can be set by: (1) the DRACeo UI or (2) automatically by an operation center function. Furthermore, the user must also specify the microservice size in terms of disk usage and serialization (i.e. the amount of data that would be sent over the network if the microservice is moved or duplicated). This attribute allows analyzing the cost of operations in terms of efficiency and energy. For example, a user can configure the deployment of a microservice (setting up resources consumption: CPU (ghz), RAM (MB), network (MB/s), HDD (MB/s) and size (MB)) to start running after $\mathrm{X}$ minute after simulation start.

4) Microservices suppression: Similar to deployment, a microservice can be terminated/killed by using a simulator UI button, or according to a defined amount of time (also set using the UI). The microservice will simply stop using the device's resources and will no longer be available for any item.

5) Microservices migration: DRACeo can move microservices between devices. This can be done from the operations center or from a specific device, which knows its connected peers and is able to execute decentralized algorithms.

When a microservice is moved, firstly it releases the resources of its current device. Then, DRACeo simulates the moving process by using the involved network connection for a period of time based on the microservice size. Finally, it starts to compete for the resources of the new device. If a microservice is moved and becomes unreachable for one of its dependencies, the user will be able to specify search mechanisms for a duplicate instance or establish special search and path optimization mechanisms to solve the problem.

6) Microservices duplication: DRACeo knows each microservice's function. Thus, it is also capable of duplicating microservices when, for example, the scheduling algorithm decides that a microservice is very requested and its overload produces excessive energy consumption. Thus, a user or an algorithm can specify where to duplicate this microservice: (1) on the same device as the original microservice, or (2) on another connected or reachable device.

7) Microservices and devices Start/Stop: DRACeo allows in a scheduled or manual way to (re)start/stop devices or microservices. Both also operate as deployment and suppression of devices and microservices. The only difference is that both elements do not disappear permanently. This allows generating scenarios based on energy saving techniques such as putting into sleep mode unused devices [21].

8) QoS definition: DRACeo allows defining any QoS heuristics, either at microservice or application level. However, the simulator offers two default heuristics: (1) "nondependent" and (2) "dependent" approach. For the first one, DRACeo, calculates the QoS value of the microservices in proportion to the hardware resources needed-obtained; while the QoS of the application is also obtained proportionally to the QoS of each microservice that composes it. Thereby:

- For a microservice $M$ being executed in a device $D$, a DRACeo user can define the ideal quality of (service QoS $=100 \%$ ) by specifying: (1)the resources that $M$ requires to run in terms of CPU frequency in $\mathrm{GHz}, \mathrm{RAM}$ consumption in $\mathrm{MB}$, network transfer rate in $\mathrm{MB} / \mathrm{s}$ and hard disk transfer rate in $\mathrm{MB} / \mathrm{s}$ and (2) an impact value that each of these needs has. These last value must be considered from $0-1$ and its sum must be 1 .

- Then, there might be some differences between the resources $M$ required to run and what it gets from $D$. Those differences are expressed in percentage (from 0-100) for each difference: (1) $D_{C}$ CPU frequency, (2) $D_{R}$ for RAM, (3) $D_{N}$ for network transfer rate and (4) $D_{H}$ for hard disk transfer rate. Thus, a microservice QoS is defined by:

$$
Q o S_{M}=I_{1} D_{C}+I_{2} D_{R}+I_{3} D_{N}+I_{4} D_{H} \text { where } \sum_{i=0}^{4} I_{i}=1 \text { (1) }
$$

Similarly, the $Q o S$ of an application $Q_{o} S_{a p p}$ is defined by the QoS of each of the $N$ microservices $Q o S_{i}$ that compose it, based on the impact value $I_{i}$ that the user defines.

$$
\sum_{i=0}^{n} \operatorname{QoS}_{i} * I_{i} \text { where } \sum_{i=0}^{n} I_{i}=1
$$


On the other hand, DRACeo also offers a "dependent" heuristic, in which the $Q o S$ of a microservice $M$ is limited by the least satisfied demanded resource, taking into account the impact parameter I specified by the user:

$$
Q o S_{M}=\operatorname{Min}\left(I_{C} D_{C}, I_{R} D_{R}, I_{N} D_{N}, I_{H} D_{H}\right)
$$

Then, the $Q o S$ of an application $A P P$ composed by a set of microservices $M=\left[M_{0} \ldots M_{n}\right]$ is limited by the microservice $M_{i} \in M$ with the lowest $Q o S$ value:

$$
\operatorname{QoS}_{A P P}=\operatorname{Min}\left(Q o S_{M_{i}}\right) \forall M_{i} \in M
$$

9) QoS analysis: Our simulator can evaluate the QoS of applications and microservices at a specific rate defined by the user when a scheduling algorithm is being executed. Operations such as graphical PLOT, data storage and linear/polynomial regression are supported.

10) Energy consumption parameters definition: For DRACeo, power consumption is based on the usage level of each component of each device. Then, the user can specify the formula of his choice for each device to relate the CPU frequency used, and the current transfer rates of the hard disk and the network (RAM consumption is independent of its load), with the power consumption expressed in Watts. This dynamism allows the user to display specific parameters of each component model, normally specified in the datasheets. However, by default, DRACeo specifies the following models, both to measure the energy of devices and microservices:

- CPU energy measurement: CPU [22] power consumption can be understood in terms of its capacitance $C$, voltage $V$ and frequency $F$.

$$
P=C V^{2} F
$$

Then, the user must specify $C, V$ and $F$ to measure the current CPU energy consumption. Note that to measure the CPU consumption of a microservice $M, F$ must be equal to $M^{\prime} s$ frequency, assuming that $M$ is the only current process in the device.

- NIC and HDD energy measurement: From several works which study energy consumption [23] [24] [25] [26] [27] [28] [29], we have observed that: (1) there is a direct relation between the transfer rates and energy consumption and (2) both HDDs and NICs have different consumption rates when they send/receive data and when they are in idle state. For this reason, we have modeled the energy consumption $E$ of both components in the same way. We established a $W_{u}$ and a $W_{i}$ value for each device which represent the consumption in watts when its NIC or HDD is in active state and the consumption in watts when it is in idle state, respectively. For a microservice $M$ with a transfer rate $L_{i}, W_{u}$ will multiply $L_{i}$ relative to the maximum transfer rate capacity $L_{M A X}$. Then, this result will be added to the idle state consumption. For this, $W_{i}$ will multiply the complement of the current component load $L$ (i.e. $L_{M A X}-L$ ) relative to $L_{M A X}$. We consider that the idle state that $M$ generates in the component, is proportional in the same way that $L_{i}$ is for $L$.

$$
E=W_{u} \frac{L_{i}}{L_{M A X}}+W_{i} \frac{L_{M A X}-L}{L_{M A X}} * \frac{L_{i}}{L}
$$

Note that to find the component's power consumption, instead of for a microservice, $L_{i}=L$

11) Energy consumption analysis: DRACeo also allows measuring the energy consumption in a time range. This analysis can be done for each device or for the entire scenario (all devices). Operations such as graphical PLOT, data storage and linear/polynomial regression are supported.

12) Centralized/non-centralized scheduling algorithm Start/Stop: When DRACeo executes a scheduling algorithm, it automatically displays metrics necessary for performance analysis: (1) Run time, (2) number of operations performed, (3) data transmitted by movements/duplications on device or across the network, (4) energy used for movements/duplications on device or across the network and (5) load per device and global load.

13) Save/Load: Deployments and execution stages.

\section{RESUlTS}

In order to test the effectiveness of DRACeo, we implemented two "naive" approaches (centralized and noncentralized) for energy savings. The objective here, is to validate that we can deploy any type of energy saving scheduling approach and then, perform its analysis in terms of QoS.

\section{A. Approach $1:$ Decentralized}

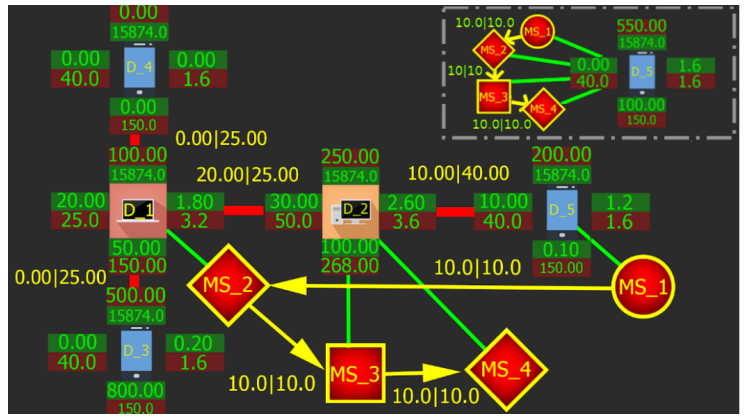

Fig. 1. Sample decentralized-mixed network

The network of figure 1, is managed by DRACeo according to equations described in section III-B for energy management/evaluation and for QoS calculation. We deployed 5 connected devices with capacities shown in the outline of each one in terms of used/available network (left: MB/s), RAM(top: $\mathrm{MB}), \mathrm{CPU}($ right: Ghz) and hard drive rate(down:MB/s). Initially (Top right of figure 1) D_5 is running an application composed of 4 differents (i.e. GUI and "control" CPU intensive for this example) microservices which have requirements in terms of [CPU-Ghz,RAM-MB, network-MB/s,HDD-MB/s, size-MB]: (1) GUI: MS_1 [1.2,200,0,0.1,50], (2)Control MS_2 $[1.8,100,0,50,50],(3)$ Control MS_3 [0.8,150,0,50,50], (4)Control MS_4 [1.8,100,0,50,50]. Then, every 1 to 5 seconds each supervisor checks energy consumption of its device against an arbitrarily threshold. If exceeded, the device will try to move the heaviest microservice to the freest neighbor. 
At runtime, since this approach has no end and is quite naive, it makes the microservices MS_2, MS_3 and MS_4 oscillate between devices D_1, D_2, D_3 and D_4. However DRACeo is able to store the best iteration. It found that the best deployment is the one represented in figure 1, where the microservices that saturated the CPU of the smartphone D_5 are running now on the laptop D_1 and the PC D_2. Moreover in figure 2, we note an initial low power consumption since only D_5 resources are used. Then, it has increased considerably, because now the network cards of D_1, D_2 and D_5 are saturated and the D_1 and D_2 CPU, RAM and transfer rate have increased their load as well (note that: (1)microservices now use the network to communicate to each other and (2) in this particular case, since no direct connections between D_5 and D_1 exist, microservices 1 and 2 use D_2 as a connection path, saturating its network card). The prototype interprets this deployment as an ideal one, because it obtains highest QoS (i.e. $100 \%$ ) and lowest possible consumption (i.e. 97,8 watts). Furthermore, to obtain this solution, DRACeo ran the algorithm for 43.8 seconds, performing 148 microservices movements between all devices, which means $7400 \mathrm{MB}$ transmitted and $0.0036 \mathrm{kWh}$ consumed.
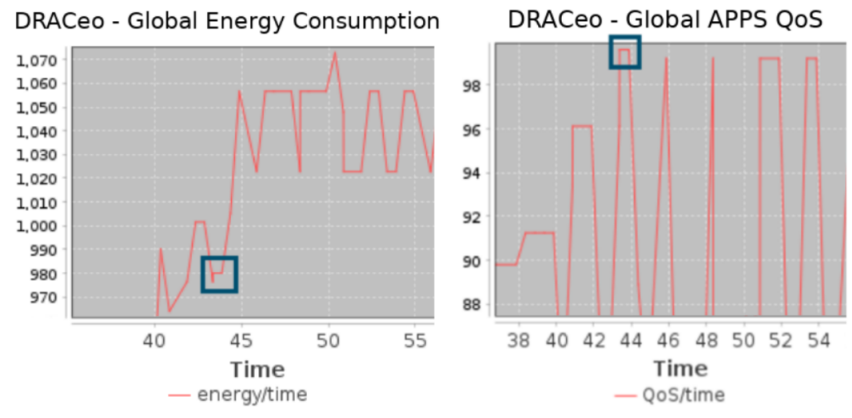

Fig. 2. Results of non-centralized algorithm evaluation

DRACeo is also capable of showing metrics for each device. For instance, D_4 was the device least involved in the execution of the algorithm, performing only 16 operations which means $800 \mathrm{MB}$ transmitted and an energy use of $0.000016 \mathrm{kWh}$.

We can conclude for example that, if we apply naive distributed scheduling algorithms like this, we can get some interesting deployment proposals in which there is an inverse relation between QoS and energy consumption. However, it lacks efficiency and predictability in terms of the amount of time and resources invested to reach a solution.

\section{B. Approach 2 : Centralized}

The network of figure 3 has the same settings as the previous example for energy management/evaluation, QoS calculation and resource view. In this second approach, a single device (in fact, a server) executes the scheduling algorithm. Every 5 seconds, it monitors the load of the rest of devices and checks that they do not exceed an arbitrary threshold. If this happens, it will try to move the most expensive microservice from the affected device to the freest one. Initially, (bottom left of figure 3) D_2 is running an application composed of 4 microservices with requirements in terms of [CPU-Ghz,RAM-MB, network-MB/s, HDD-MB/s, size]: GUI MS_1 [1.2, 200, 0,0.1, 50], Control: MS_2 [1.8,100,0,0.1,50], Control: MS_3 $[0.8,150,0,100,50]$ and Control: MS_4 [1.8,100,0,0.1,50].

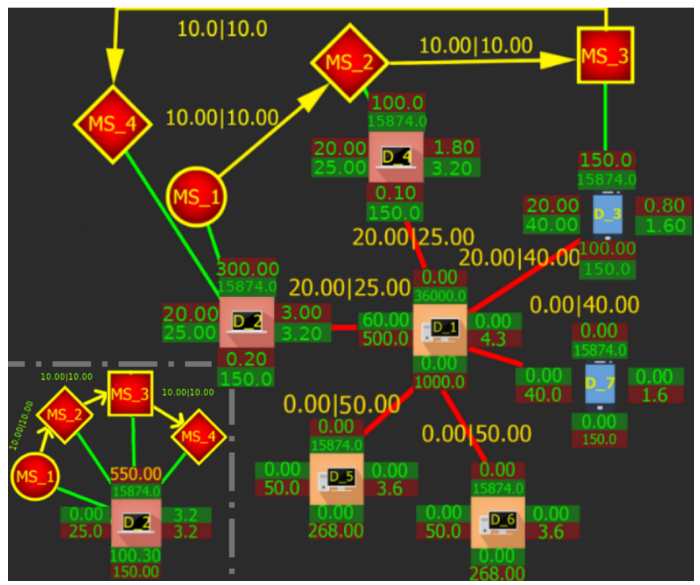

Fig. 3. Sample mixed-centralized network

At runtime, $D_{1}$ tries to move the heaviest microservice to the node with least load (except MS_1 because it manages a GUI process). Similar to the previous example, it makes the microservices MS_2, MS_3 and MS_4 oscillate between the other peers. However, unlike the previous algorithm, this algorithm finds a solution and stabilizes in a single deployment shown in figure 3, where the microservices that saturated the resources of D_2 are running now on D_4 and D_3. In addition, in figure 4, we can see that the overall power consumption at the beginning was low since only the resources of device D_2 are used. Then, it has increased because now, the resources of D_3 and D_4 are being used.
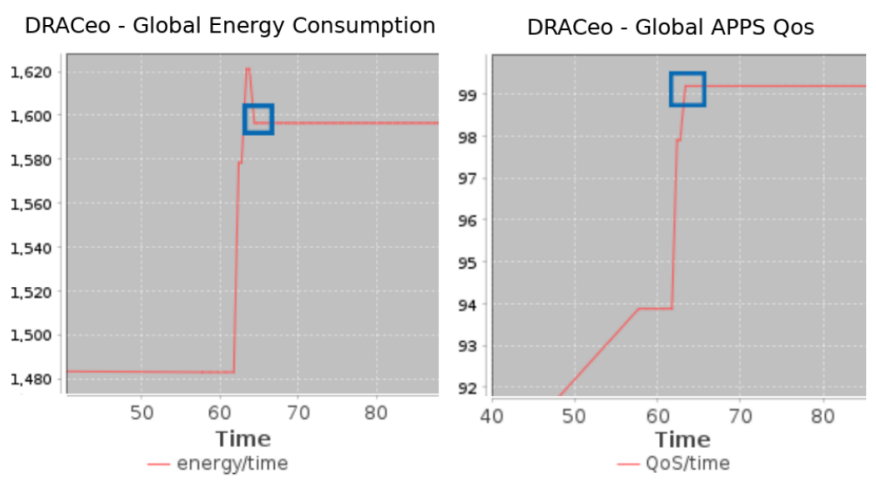

Fig. 4. Results of centralized algorithm evaluation

The simulator interprets that this is the best deployment since it obtains the highest QoS (i.e. $100 \%$ ) and the lowest possible energy use (159,8 watts). Furthermore, to obtain this solution, DRACeo ran the algorithm for 64.3 seconds, performing 70 microservices movements, which means $3500 \mathrm{MB}$ transmitted and an energy use of $0,00018207 \mathrm{kWh}$.

It is interesting to see that both approaches, which managed similar applications, have different advantages and disadvantages. For example, the first one reaches a solution 20.5 
seconds before the second one; but the second one performs fewer operations, which means $3900 \mathrm{MB}$ in operations transmissions and $0,00341793 k W h$ saved. On the other hand, the first one is more unstable than the second (which is linear from the second 64); but it offers more variety of deployment options by time interval. These two observations, for example, allow us to understand some characteristics of centralized and decentralized distributed approaches like those, where either with a greater number of nodes and microservices, the former will offer more stable computational behavior and a controlled number of operations; while the latter will offer greater diversity of solutions in less time.

As we see, DRACeo can deploy both approaches (or more complex ones [7]), allowing the analysis of several variables that describe the defined QoS, whole or per device energy consumption, whole or per device amount of data transmitted and the time to get interesting results.

\section{COnClusions AND FUture Works}

In this work, we have presented our simulator called DRACeo. It is capable of deploying and managing any type of network and heterogeneous devices to run distributed applications based on services or microservices. DRACeo implements functions of (un)deploying dynamically devices and deploying, deleting, moving and duplicating microservices to allow performing centralized and non-centralized planning algorithms. At run time, the simulator is capable of monitoring several variables that allow understanding the efficiency of the technique deployed: Execution time, amount of energy spent, current quality of service and amount of data transmitted are some good examples. It is important to say that in order to find the value of QoS and energy consumption, the simulator allows users (defaults approaches are provided) to define their own heuristics or formulas. The objective of DRACeo is to allow testing dynamic deployment scheduling algorithms that redeploy microservices in order to save energy while conserving a certain QoS. Thereby, DRACeo will help developers and researchers find the best deployment and best distribution behavior in any network of heterogeneous devices.

Actually, we are improving the scalability of DRACeo, taking into account discrete and deterministic approaches as well as real deployments of microservices and devices. On the other hand, we are updating the heuristics to determine the power consumption in some hardware components with special features(CPU turbo boost, DVFS, etc).

\section{REFERENCES}

[1] A. W. Services, "Implementing microservices on aws," vol. 2019, 2019.

[2] DOCKER, "Debug your app, not your environment," https://www.docker.com/, 2020

[3] K. Da, M. Dalmau, and P. Roose, "Kalimucho: Middleware for mobile applications," in Proceedings of the 29th Annual ACM Symposium on Applied Computing, ser. SAC '14, 2014.

[4] NETFLIX, "Netflix conductor: A microservices orchestrator," https://netflixtechblog.com/netflix-conductor-a-microservicesorchestrator-2e8d4771bf40, 2016.

[5] H. H. lvarez Valera, P. Roose, M. Dalmau, C. Herzog, and K. Respicio, "Kaligreen: A distributed scheduler for energy saving," Procedia Computer Science, 2018.
[6] H. H. lvarez Valera, M. Dalmau, P. Roose, and C. Herzog, "The architecture of kaligreen v2: A middleware aware of hardware opportunities to save energy," in 2019 Sixth International Conference on Internet of Things: Systems, Management and Security (IOTSMS), 2019.

[7] E. Bongers and J. Pouwelse, "A survey of p2p multidimensional indexing structures," 2015.

[8] packetstorm, "Network simulation," https://packetstorm.com/networksimulation/, 2018.

[9] G. C. Inc., "Ip wan emulator," https://www.gl.com/wan-link-emulationipnetsim.html, 2020.

[10] CISCO, "Cisco packet tracer," https://www.netacad.com/courses/packettracer, 2020

[11] Greencloud, "Greencloud - the green cloud simulator," https://greencloud.gforge.uni.lu/, 2017.

[12] S. Sundresh, Wooyoung Kim, and G. Agha, "Sens: a sensor, environment and network simulator," in 37th Annual Simulation Symposium, 2004. Proceedings., 2004, pp. 221-228.

[13] S. Agrawal, S. Shailendra, B. Panigrahi, H. K. Rath, and A. Simha, "Oicn simulator (oicnsim): An ns-3 based simulator for overlay information centric networking (o-icn)," in Proceedings of the 1st Workshop on Complex Networked Systems for Smart Infrastructure, 2018.

[14] cloudbus, "Containercloudsim: An environment for modeling and simulation of containers in cloud data centers," http://www.cloudbus.org/cloudsim/container.html, 2016.

[15] Z. Nikdel, B. Gao, and S. W. Neville, "Dockersim: Full-stack simulation of container-based software-as-a-service (saas) cloud deployments and environments," in 2017 IEEE Pacific Rim Conference on Communications, Computers and Signal Processing (PACRIM), 2017.

[16] Y. Zhang, Y. Gan, and C. Delimitrou, " $\mu q$ sim: Enabling accurate and scalable simulation for interactive microservices," IEEE International Symposium on Performance Analysis of Systems and Software (ISPASS), 2019.

[17] H. Gupta, A. Vahid Dastjerdi, S. K. Ghosh, and R. Buyya, "ifogsim: A toolkit for modeling and simulation of resource management techniques in the internet of things, edge and fog computing environments," Software: Practice and Experience, 2017.

[18] P. Novotny and A. Wolf, "Simulating services-based systems hosted in networks with dynamic topology," 2016.

[19] A. D. M. D. Esposte, E. F. Santana, L. Kanashiro, F. M. Costa, K. R. Braghetto, N. Lago, and F. Kon, "Design and evaluation of a scalable smart city software platform with large-scale simulations," Future Generation Computer Systems, 2019.

[20] IBM, "Inside the linux 2.6 completely fair scheduler," https://developer.ibm.com/technologies/linux/tutorials/l-completelyfair-scheduler/, 2018.

[21] N. M. Azmy, I. A. El-Maddah, and H. K. Mohamed, "Adaptive power panel of cloud computing controlling cloud power consumption," in Proceedings of the 2Nd Africa and Middle East Conference on Software Engineering, 2016.

[22] Intel, "Enhanced intel speedstep technology for the intel pentium $\mathrm{m}$ processor," 2004.

[23] S. Kiertscher and B. Schnor, "Scalability evaluation of an energyaware resource management system for clusters of web servers," in Proceedings of the International Symposium on Performance Evaluation of Computer and Telecommunication Systems, ser. Spects '15, 2015.

[24] K. Zhan, C.-H. Lung, and P. Srivastava, "A green analysis of mobile cloud computing applications," in Proceedings of the 29th Annual ACM Symposium on Applied Computing, 2014.

[25] A. Orgerie, L. Lefèvre, I. Guérin-Lassous, and D. M. Lopez Pacheco, "Ecofen: An end-to-end energy cost model and simulator for evaluating power consumption in large-scale networks," in IEEE International Symposium on a World of Wireless, Mobile and Multimedia Networks, 2011.

[26] B. F. Cornea, A. Orgerie, and L. Lefèvre, "Studying the energy consumption of data transfers in clouds: the ecofen approach," in 2014 IEEE 3rd International Conference on Cloud Networking (CloudNet), 2014.

[27] S. Chiaravalloti, F. Idzikowski, and L. Budzisz, "Power consumption of wlan network elements," 2011.

[28] Segate, "Desktop hdd product manual," https://www.seagate.com/wwwcontent/product-content/barracuda-fam/desktop-hdd/barracuda-720014/en-us/docs/100686584v.pdf, sep 2016.

[29] Samsung, "Samsung v-nand ssd 860 evo," dec 2017. 